\title{
THE EXPLOSIVE GROWTH OF GRAPH THEORY
}

\author{
Frank Harary \\ Department of Mathematics \\ University of Michigan \\ Ann Arbor, Michigan 48109
}

The preparation of an article that documents the remarkable growth of research activity in the theory of graphs during the past few decades was suggested to me by the important commentary of May [12] on the growth and quality of mathematical literature.

\section{BOOKS AND CONFERENCE BOOKS}

The first book on graph theory as a separate subject in mathematics was written by König [11] in 1936. He wrote in German because he correctly decided that if it were written in his native Hungarian, very few people would be able to read it. This book was reprinted in the United States in 1950. When I first became interested in graph theory in September 1950, this was the only book available on the subject. There were some theorems on graphs in the pioneering book on topology by Veblen [15] whose first chapter takes up "linear graphs." There were also some booklets by Lucas and Sainté-Lague, referenced in König's book, on the use of graphs in solving puzzles and in recreational mathematics. There were in 1950 literally only about a dozen mathematicians in the world actively doing research in graph theory!

The second book on graph theory, by Berge [1], appeared in French in 1958. I had begun working on a book on graph theory since 1950, virtually the moment I was exposed to this fascinating aesthetic art form. As I was writing each chapter, so many tractable open questions suggested themselves that research impeded the process of writing. For this reason, my book [10] did not appear until 1969. CHART 1 displays the number of books which dealt with either graph theory as a separate subject, some special topic in graph theory, or a particular application of graph

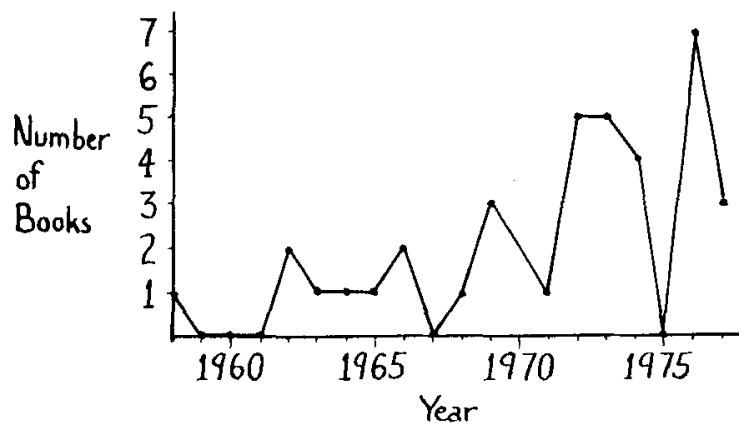

Chart 1. Books devoted wholly or in part to Graph Theory. 


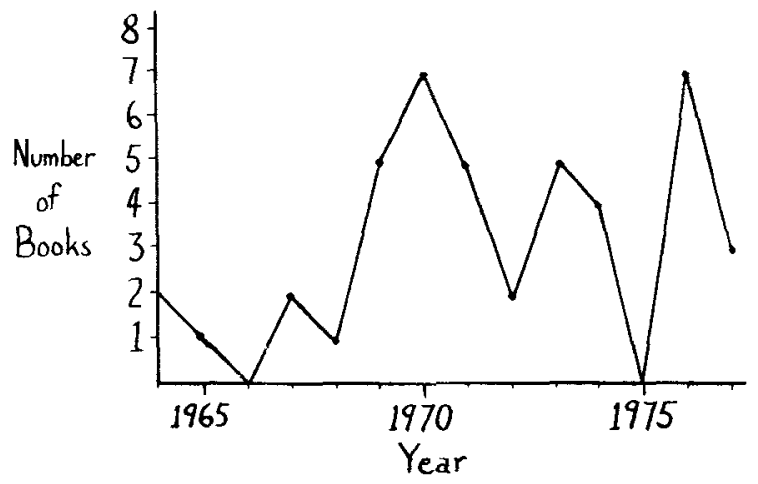

Chart 2. Proceedings of conferences devoted wholly or in part to Graph Theory.

theory to another science: physical, social, or engineering, beginning with 1958 (it is called a chart because I cannot call it a graph).

There is another important outlet for research in graph theory in book form. These are the "conference books," shown in CHART 2, which report the proceedings of symposia on graph theory, combinatorial theory, and their applications. The first of these conference books, to my knowledge, was the book [8] of the Proceedings of the First Czechoslovakian Symposium on Graph Theory held in Smolenice in 1963.

In constructing CharTs 1 and 2 , it was a matter of subjective judgment as to how much graph theory should be adequate for inclusion. When a book has been translated, only the original was included. When a conference book appeared in more than one volume, it was still counted as just one book. When there was more than one printing of a book, it was included as just one book. A list of books and conference books which includes most of those reported in CHARTS 1 and 2 appeared in the Graph Theory Newsletter [9].

\section{Published Papers on Graph Theory}

In ancient times, articles on graph theory were reviewed in Mathematical Reviews $(\mathrm{MR})$ in one of the following categories:

(1) Foundations, Theory of Sets, Logic

(2) Algebraic Topology

(3) Geometry

(4) Numerical Analysis

(5) Probability

(6) Statistics

(7) Physical Applications, in the subsection on Optics, Electromagnetic Theory, Circuits

(8) Other Applications, in the subsections on Economics, Management Science, Biology and Sociology, Information and Communication Theory.

Even though most papers on the subject were reviewed under Algebraic Topology (at the end of the section on Topology), in general the papers were neither algebraic nor topological, but combinatorial or as I would prefer to express it, graphical. 
The first mention of Combinatorial Analysis as an area of mathematics in MR was in Volume 20, Number 1, January 1959. But Graph Theory (GT) was not yet mentioned. Papers on graph theory were reviewed in this new category as well as in the eight sections listed above. It wasn't until 1968 that MR in Volume 35 stated Category 05 as "Combinatorial Theory, Graph Theory." Mysteriously, the beautiful words "Graph Theory" then disappeared from the front cover of MR beginning with the July 1973 issue, Volume 46, when the name of 05 was changed to "Combinatorics." It would be splendid if this could be altered again so that 05 reads "Combinatorics, Graph Theory" and this would improve the front cover of MR.

Since its establishment, the number of papers in Category 05 has been increasing exponentially, far more rapidly than the number of papers in all branches of mathematics. There have been so many papers in Category 05 in recent years that one could even use the number of pages in MR as an indication of the growth rate of publication. Graph Theory is now the subcategory $05 \mathrm{C}$.

The number of graphical papers reviewed in Volume 20,1959, of MR was only seven. In fact, my review of Berge's book appeared in MR, Volume 21, under Algebraic Topology.

Beginning in 1973, MR launched in Volume 45 the extremely useful inclusion in its Index issues of the names of authors within the subject index. A remarkably convincing CHART 3 which displays the recent exponential growth in $05 \mathrm{C}$ can be constructed by estimating accurately, to the nearest fourth of a column. the number of columns in the author list in the index issue. This is shown using solid lines.

The subcategory $94 \mathrm{~A} 20$ includes papers on applications of graph theory. When the number of columns from this source is added to the number for $05 \mathrm{C}$, the result is shown in CHART 3 as dotted lines; it also has an exponential appearance. Unfortunately, MR decided to change this format in Volume 53 so that CHART 3 only reports Volumes $45-52$.

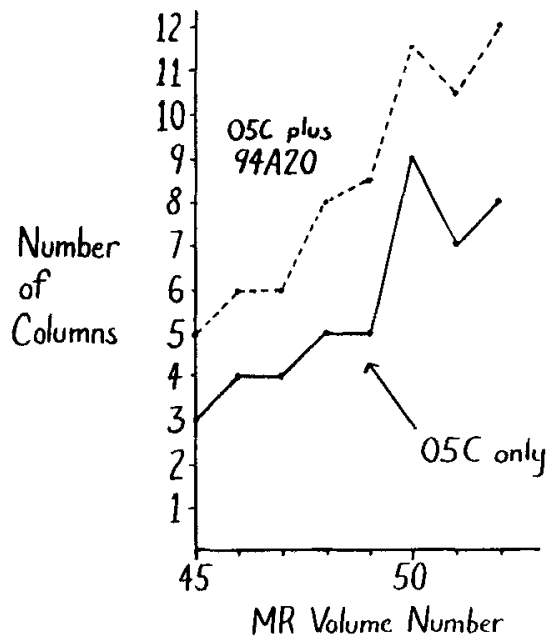

Chart 3. Number of columns devoted to Graph Theory in MR Subject Index. 


\section{JOURNALS}

The first mathematical journal devoted to combinatorics and graph theory began publication with Volume 1 in 1966. This was the Journal of Combinatorial Theory (JCT) which was founded by G.-C. Rota and myself as a result of exploratory initial discussions in Frascati during July 1964 over much Italian wine and pasta. It began as a quarterly and almost immediately acquired a three-year backlog of papers. In 1970, it was separated into two parts which are easily described in reverse alphabetical order: Series B comprises Graph Theory and Matroids; Series A includes all other areas of combinatorics. Since then, JCT has been a monthly.

The second such journal was Discrete Mathematics (DM) which was founded by Peter Hammer. It also began as a quarterly with Volume 1 having its first three issues in 1971 and its fourth in early 1972. With four issues comprising each volume, DM in 1973 became a monthly periodical having three volumes per year, with only one exception in 1974. That year DM had four volumes as a result of a special report on coding theory. Its plans are to remain a monthly indefinitely. However, in order to cope with its backlog, it will soon increase the number of pages per issue.

The journal DM has recently given birth to a series of books titled Annals of Discrete Mathematics. These resemble somewhat the Springer Lecture Notes in Mathematics, of which Volumes 110,186, 303, 406, 411, and 642 report proceedings of combinatorial conferences with a primary emphasis on graph theory. In addition DM has another spin-off: a new journal, Discrete Applied Mathematics, with Volume 1 in 1979.

There are other publications involving graph theory which merit mention here. The informal and informative, but important, Graph Theory Newsletter was founded at Western Michigan University largely influenced by Gary Chartrand. It began in 1971 and has as its basic purpose the rapid, relatively inexpensive dissemination of interesting information to graph theorists all over the world. It includes abstracts of results, announcements of forthcoming meetings, lists of recently published papers by title, and lists of books. More recently it has been presenting short expository articles.

In the field of applications, the journal Networks was launched in 1972, founded by I. T. Frisch and A. B. Frank. By definition, a network consists of a graph (or a digraph, a multigraph, or other generalization) together with values assigned to its edges or its vertices, or both). Of course networks are directly involved with real world problems involving time and money, while graph theory constitutes the basic mathematical model underlying networks.

Other active applications using GT are in the fields of electrical networks, operations research and computer science. The journals in these fields often publish articles on either so-called "pure" graph theory or its applications to the respective fields. These journals include, amongst many others:

Transactions of the IEEE (Institute for Electrical and Electronic Engineering) on CAS (Circuits and Systems), Operations Research, Journal of the ACM and

Communications of the ACM (Association for Computing Machinery), SIAM

(Society for Industrial and Applied Mathematics) Journal on Computing.

In fact, "graph theory" is a subject in the classification schema of both Physics Abstracts and Chemical Abstracts.

In the applications of graph theory to the social sciences, relevant articles have appeared in the journals: 
Behaviorial Science, Journal of Mathematical Sociology, Psychometrika, Social Networks, Sociometry.

The year 1976 saw a noticeable increase in combinatorial journals as it was the first year for both Ars Combinatoria and the Journal of Combinatorics, Information and System Sciences.

The Journal of Graph Theory (JGT) began in 1977. Of the 10,000 new journals in the professional and scientific fields which were launched worldwide that year, JGT was voted by the Association of American Publishers (Technical, Scientific and Medical Division) as being the most outstanding new journal of 1977.

\section{BibliograPhiEs OF GRAPH TheORY}

The first really comprehensive bibliography of GT was the list of references up to 1936 in the book by König. Very recently, the first book on the history of graph theory by Biggs et al. [6] pointed out several additional appropriate references dating before 1936. Together, these two sources provide an admirable bibliography of early works on GT.

The next few listings appeared in books on GT including those by Berge [1], myself [10], and many others (see [9]). But these cannot be considered comprehensive because they were not, as they reflected the authors' subjective criteria for inclusion.

The genuine bibliographies of graph theory which have appeared to date are those compiled by Turner [13], Deo [7], and most recently, by Berman $[2,3,5]$ of works through the middle of 1973 . The latter proved particularly useful as the articles in it enabled Berman [4] to develop citation indexes for papers and books on GT.

Turner and Katz [14] compiled an informative annotated bibliography of research articles on graph theory in the Soviet Union to 1969. There has been a notable further increase in graph theory and its applications in the Russian literature during the past decade and it is now time to recommend that a sequel to [14] be continued on a regular basis. It would appear quite safe to predict that we shall soon see an awakening to the importance of graph theory for applications to the real world in the Chinese research literature.

Together, these bibliographies provide dramatic evidence of the explosive growth of graph theory.

\section{SYMPOSIA}

There have been many national and international symposia on graph theory, combinatorial theory, and their applications. These have taken place intermittently in Canada, Czechoslovakia, France, Germany, Great Britain, Hungary, Italy, and the United States. The author has had occasion to speak at conferences on the applications of graph theory to the following topics: electrical networks, management sciences, social sciences, architecture, linguistics, theoretical physics, operational research, linear programming, sparse matrices in numerical analysis, information retrieval, biology, design problems in engineering, didactics, social networks, peace science, theoretical chemistry, and theoretical computer science. Whenever such an invitation materializes, I take the point of view that the organizers of the conference are aware of the fact that the only subject I know in some detail is GT. Hence 
I ask them what aspects of GT are the most relevant and applicable to their fields and prepare my lectures accordingly.

During my world-wide travels, I have lectured not only at colloquia in departments of mathematics at various universities but also in the following other departments:

Applied mathematics, statistics, operations research, civil engineering, architecture, psychology, physics, chemistry, electrical engineering, computer science, combinatorics and optimization, anthropology, sociology, and Australian environmental studies.

This remarkable variety of subjects attests vividly to the essentially universal applicability of the theory of graphs as a useful and valid mathematical model.

\section{Conclusion and Prediction}

Graph Theory is a very dynamic subject: some areas are slowing down (attempts to prove the Four Color Conjecture), others are growing (ramsey theory, reconstruction conjecture), and new ones are being born (isomorphic factorizations, multiple interval graphs, coloring digraphs). The most established scientific areas in which GT is regularly used as a mathematical model are electrical engineering and theoretical chemistry. In fact, a series of papers titled "The graph-like state of matter" is being written by $\mathrm{M}$. Gordon et al. In the social sciences, the most recent application areas are anthropology, sociology, and political science. Several underdeveloped subjects are also beginning to use GT.

The evidence is uncontrovertible that the present growth status of graph theory and its applications is genuinely explosive. My prediction is that we are now witnessing only the early beginnings of this phenomenon and that in time the importance of discrete mathematics including the fields of matroids and networks, and of GT in particular, will become comparable to the present scope of the more traditional applied mathematics.

\section{SUMMARY}

The growth of the literature in graph theory is described in several ways: (1) by graphing the number of books on the subject per year, (2) by analyzing the number of published papers as reported in Mathematical Reviews, (3) by displaying the growth of journals whose primary emphasis is on graph theory and combinatorial theory, and (4) by noting various bibliographies on graph theory. The observations lead to the conclusion that the literature of graph theory is growing explosively.

\section{REFERENCES}

1. Berge, C. 1958. Theorie des Graphes et ses Applications. Dunod, Paris.

2. Berman, G. 1975. Forward Citations in Graph Theory. University of Waterloo. Waterloo, Canada.

3. Berman, G. 1977. Applied Graph Theory Bibliography with Forward Citations. University of Waterloo, Waterloo, Canada. 
4. Berman, G. 1977. Frequently cited publications in pure graph theory. J. Graph Theory $1: 175-180$.

5. Berman, G. \& D. M. JACKson. 1976. Forward Citations in Enumerations. University of Waterloo, Waterloo, Canada.

6. Biggs, N., K. LloYd \& R. Wilson. 1976. Graph Theory 1736-1936. Oxford University Press, Oxford.

7. Deo, N. 1969. An extensive English language bibliography on graph theory and its applications. Jet Propulsion Laboratory, Pasadena, Technical Report (October); Supplement 1 (April 15, 1971).

8. Fiedler. M.. Editor. 1964. Theory of Graphs and its Applications. Academia. Prague.

9. Graph Theory Newsletter, Supplement: A List of Books in Graph Theory and Related Areas 2(197), No. 4.

10. Harary, F. 1969. Graph Theory, Addison-Wesley, Reading, Mass.

11. KöNIG, D. 1936. Theorie der endlichen und unendlichen Graphen. Leipzig: Reprinted Chelsea, New York (1950).

12. MAY, K. O 1968/69. Growth and quality of the mathematical literature. Isis, 59: 363-371.

13. Turner, J. 1969. Key-word index bibliography of graph theory. Proof Techniques in Graph Theory. F. Harary, Ed. Academic Press, New York, N.Y. pp. 189-330.

14. Turner, J. \& W. H. Katz. 1970. A survey of progress in graph theory in the Soviet Union. SIAM Review (Supplement). 12: 1-68.

15. Veblen, O. 1922. Analysis Situs. Amer. Math. Soc. Colloq. Publ. Vol. 5. Cambridge. (2d edit. New York. 1931). 